\title{
Obtenção do módulo dinâmico de misturas asfálticas com agregados sintéticos de argila calcinada, utilizando o ensaio de compressão diametral
}

\author{
Fábio Roberto Garcia Nunes ${ }^{1}$; Cleudinei Lopes da Silva²; Consuelo Alves da Frota ${ }^{3}$
}

\begin{abstract}
Resumo: No Brasil, o módulo dinâmico de misturas asfálticas ainda é pouco utilizado, sobretudo por ser, em regra, obtido por intermédio do estado de compressão ou da tração axial, a necessitar, em razão disso, de corpos de prova com aproximadamente $200 \mathrm{~mm}$ de altura, a partir de um compactador giratório. Tal imperativo dificulta os estudos realizados com amostras retiradas diretamente do pavimento. Neste trabalho, determinou-se o citado parâmetro por meio do ensaio de compressão diametral em corpos de prova compactados (Marshall) de misturas asfálticas, nas dimensões 101,5 × 63,50 mm. Figuraram como agregados graúdos os agregados sintéticos de argila calcinada (ASAC). Os resultados se mostraram equivalentes entre as misturas asfálticas com ASAC e segundo o ensaio de compressão diametral com aqueles obtidos pelo trabalho de KING (2004), quando utilizado o ensaio de compressão axial.
\end{abstract}

Abstract: In Brazil, the asphalt mixture dynamic modulus is still little used, because it requires $200 \mathrm{~mm}$-high test samples from a gyratory compactor. Such requirement makes it difficult to carry out studies on samples removed directly from the pavement. In this work, the dynamic modulus has been determined by diametral compression test in Marshall samples with standard dimensions $(101,5 \times$ $63,5 \mathrm{~mm}$ ). Calcinated clay synthetic aggregates (ASAC) were used in the composition of the mixture as coarse aggregates. The results showed that it is possible to achieve the dynamic modulus by means of diametral compression test and they are comparable to the obtained by KING (2004) when using the axial compression test was used.

\section{INTRODUÇÃO}

As misturas asfálticas possuem comportamento dependente da taxa de aplicação de carga ou deslocamento, sobretudo devido à participação do ligante betuminoso. Seu desempenho mecânico pode variar de elástico a viscoelástico linear, para baixas temperaturas e altas taxas de carregamento e, viscoelástico não linear a viscoplástico, para altas temperaturas e baixas taxas de carregamento (Sides et al., 1985). Deste modo, a determinação das suas propriedades viscoelásticas é de vital importância para o estudo do comportamento mecânico dessas misturas.

Diversos ensaios podem ser realizados de forma a se obter as citadas propriedades, dentre outros se pode citar: o ensaio de módulo de relaxação, o ensaio de creep estático e o ensaio com troca de freqüência. Nos ensaios estáticos (módulo de relaxação e creep) é necessário aplicar-se um carregamento ou deslocamento instantâneo (o grande problema neste tipo de experimento) e constante ao corpo de prova e, em seguida, monitorar a sua evolução. Atualmente, tais ensaios são

\footnotetext{
${ }^{1}$ Fábio Roberto Garcia Nunes, Universidade Federal do Amazonas, Grupo de Geotecnia, Manaus, AM, Brasil. (e-mail: fabiorgn@gmail.com).

${ }^{2}$ Cleudinei Lopes da Silva, Universidade Federal do Amazonas, Grupo de Geotecnia, Manaus, AM, Brasil. (e-mail: cleudineilopes@gmail.com).

${ }^{3}$ Consuelo Alves da Frota, Universidade Federal do Amazonas, Grupo de Geotecnia, Manaus, AM, Brasil. (e-mail: cafrota@ufam.edu.br).
}

Manuscrito recebido em 7/12/2008 e aprovado para publicação em $1 / 6 / 2009$. Este artigo é parte de TRANSPORTES, volume XVII, número 1, junho de 2009. ISSN: 1415-7713. aproximados por rampas de carga ou deslocamento. Especificamente, no ensaio de relaxação, a aplicação de um deslocamento instantâneo requer um equipamento extremamente robusto, tornando a utilização do ensaio de creep estático mais comum na caracterização das propriedades viscoelásticas lineares.

Por outro lado, essas propriedades também podem ser obtidas no domínio da freqüência. No caso da determinação do módulo dinâmico, aplica-se um carregamento senoidal ao corpo de prova e, verifica-se a deformação. O referido módulo é calculado por meio da relação entre as amplitudes de carregamento e deslocamento, sendo a diferença entre seus picos o ângulo de fase. No decorrer do experimento varia-se a freqüência de aplicação de carga, tendo-se, portanto, valores de módulo dinâmico alusivo a diferentes freqüências. É importante ressaltar que as propriedades viscoelásticas no domínio do tempo (módulo de relaxação), igualmente, podem ser conseguidas segundo processos matemáticos, utilizando-se as propriedades oriundas do domínio da freqüência.

\section{FUNDAMENTAÇÃO TEÓRICA}

\subsection{Estado de tensões no ensaio de compressão diametral}

O ensaio de compressão diametral consiste na aplicação de uma carga na direção vertical por meio de frisos metálicos diametralmente opostos (Figura 1). Hondros (1959), considerando um estado biaxial de tensões, obteve as tensões verticais e horizontais de acordo com as equações a seguir. 


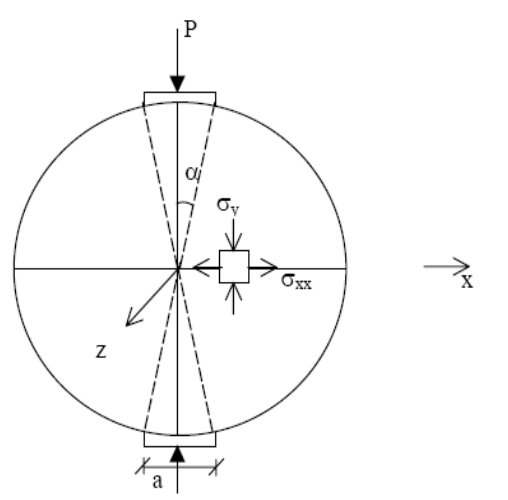

Figura 1. Esquema de montagem do ensaio (Zhang et al., 1997; Wen, 2001)

$$
\begin{gathered}
\sigma_{x}(x)=\frac{2 \cdot P}{\pi a d} \cdot\left[\frac{\left(1-\bar{x}^{2}\right) \cdot \operatorname{sen}(2 \cdot \alpha)}{1+2 \cdot \bar{x}^{2} \cdot \cos (2 \cdot \alpha)+\bar{x}^{4}} \cdots\right. \\
\left.\cdots-\tan ^{-1}\left(\frac{1-\bar{x}^{2}}{1+\bar{x}^{2}} \cdot \tan (\alpha)\right)\right]=\frac{2 \cdot P}{\pi a d} \cdot[f(\bar{x})-g(\bar{x})] \\
\sigma_{y}(x)=-\frac{2 \cdot P}{\pi a d} \cdot\left[\frac{\left(1-\bar{x}^{2}\right) \cdot \operatorname{sen}(2 \cdot \alpha)}{1+2 \cdot \bar{x}^{2} \cdot \cos (2 \cdot \alpha)+\bar{x}^{4}} \cdots\right. \\
\left.\ldots+\tan ^{-1}\left(\frac{1-\bar{x}^{2}}{1+\bar{x}^{2}} \cdot \tan (\alpha)\right)\right]=\frac{2 \cdot P}{\pi a d} \cdot[-f(\bar{x})-g(\bar{x})] \\
\sigma_{x}(y)=\frac{2 \cdot P}{\pi a d} \cdot\left[\frac{\left(1-\bar{y}^{2}\right) \cdot \operatorname{sen}(2 \cdot \alpha)}{1+2 \cdot \bar{y}^{2} \cdot \cos (2 \cdot \alpha)+\bar{x}^{4}} \cdots\right. \\
\left.\cdots+\tan ^{-1}\left(\frac{1+\bar{y}^{2}}{1-\bar{y}^{2}} \cdot \tan (\alpha)\right)\right]=\frac{2 \cdot P}{\pi a d} \cdot[m(\bar{y})-n(\bar{y})] \\
\left.\ldots+\tan ^{-1}\left(\frac{1+\bar{y}^{2}}{1-\bar{y}^{2}} \cdot \tan (\alpha)\right)\right]=\frac{2 \cdot P}{\pi a d} \cdot[-m(\bar{y})-n(\bar{y})] \\
\sigma_{y}(y)=-\frac{2 \cdot P}{\pi a d} \cdot\left[\frac{\left(1-\bar{y}^{2}\right) \cdot \operatorname{sen}(2 \cdot \alpha)}{1+2 \cdot \bar{y}^{2} \cdot \cos (2 \cdot \alpha)+\bar{x}^{4}} \cdots\right.
\end{gathered}
$$

em que, $\bar{x}=x / R ; \bar{y}=y / R ; P$ é a carga aplicada no corpo de prova; $a$ é a largura do friso de carga; $d$ é a espessura do corpo de prova; $R$ é o raio do corpo de prova; $\alpha$ é o ângulo radial.

Nota-se que as equações segundo Hondros (1959), citadas por Zhang et al. (1997) e Wen (2001), são independentes de qualquer parâmetro do material, porém, função das dimensões do corpo de prova e do friso metálico de aplicação de carga. Outra vantagem do ensaio de compressão diametral é a facilidade na realização de estudos de campo (extração de corpos de prova), visto que este tipo de experimento dispensa amostras com grandes alturas (cerca de $200 \mathrm{~mm}$ ), como no caso dos ensaios uniaxiais.

\subsection{Módulo dinâmico para o estado de compressão diametral}

Considerando-se o carregamento expresso pela equação

$$
P=P_{o} \cdot e^{i \cdot \omega \cdot t}=P_{o} \cdot[\cos (\omega \cdot t) i \cdot \operatorname{sen}(\omega \cdot t)]
$$

em que $P_{o}$ e $\omega$ são, respectivamente, a amplitude do carregamento e a freqüência angular. Considerando-se a teoria da elasticidade num estado plano de tensões e que no regime linear os materiais viscoelásticos possuem coeficiente de Poisson $v$ constante (Wen, 2001), pode-se escrever

$$
\varepsilon_{x x}=\frac{1}{E} \cdot\left(\sigma_{x x}-v \cdot \sigma_{y y}\right)
$$

Utilizando-se o módulo complexo $E=\left|E^{*}\right| e^{i \theta}$, onde $\left|E^{*}\right|$ é o módulo dinâmico e $\theta$ o ângulo de fase, e as equações 1, 2 e 5, a Equação 6 fica

$$
\varepsilon_{x x}=\frac{2 \cdot P_{o} \cdot e^{i \cdot(\omega t-\theta)}}{\left|E^{*}\right| \cdot \pi a d} \cdot(A-v \cdot B)
$$

sendo $A=f(\bar{x})-g(\bar{x})$ e $B=-f(\bar{x})-g(\bar{x})$.

Portanto, o deslocamento na direção horizontal é calculado por meio da integração da Equação 7 no espaço entre os LVDTs,

$$
U(t)=\int_{-l}^{l} \varepsilon_{x x} d x=\frac{2 \cdot P_{o} \cdot e^{i(\omega t+\theta)}}{\left|E^{*}\right| \cdot \pi a d} \cdot(\hat{A}-v \cdot \hat{B})
$$

em que: $\hat{A}=\int_{-l}^{l} A d x$ e $\hat{B}=\int_{-l}^{l} B d x$.

Observa-se que $A$ e $B$ são funções de fácil integração, com seus resultados dependendo apenas da geometria do corpo de prova e da posição dos LVDTs, podendo ser facilmente tabulados para várias geometrias e distâncias entre os LVDTs. Extraindo-se somente a contribuição senoidal e isolando-se o módulo dinâmico na Equação 8, tem-se

$$
\left|E^{*}\right|=\frac{2 \cdot P_{o} \cdot \operatorname{sen}(\omega \cdot t-\theta)}{U(t) \cdot \pi a d} \cdot(\hat{A}-v \cdot \hat{B})
$$

Seguindo-se os mesmos passos para direção vertical, tem-se

$$
\left|E^{*}\right|=\frac{2 \cdot P_{o} \cdot \operatorname{sen}(\omega \cdot t-\theta)}{V(t) \cdot \pi a d} \cdot(\hat{C}-v \cdot \hat{D})
$$

Considerando-se o material isotrópico e igualandose as Equações 9 e 10, obtém-se o coeficiente de Poisson

$$
v(t)=\frac{U(t) \cdot \hat{C}-V(t) \cdot \hat{A}}{U(t) \cdot \hat{D}-V(t) \cdot \hat{B}}
$$

Fazendo-se:

$$
U(t)=U_{o} \cdot \operatorname{sen}(\omega \cdot t-\theta)
$$




$$
V(t)=V_{o} \cdot \operatorname{sen}(\omega \cdot t-\theta)
$$

e substituindo-se as Equações 12 e 13 nas Equações 11 e 9, obtém-se:

$$
\begin{gathered}
v(t)=\frac{U_{o} \cdot \hat{C}-V_{o} \cdot \hat{A}}{U_{o} \cdot \hat{D}-V_{o} \cdot \hat{B}} \\
\left|E^{*}\right|=-\frac{2 \cdot P_{o}}{\pi a d} \cdot\left(\frac{\hat{A} \cdot \hat{C}-\hat{B} \cdot \hat{D}}{-\hat{C} \cdot U_{o}+\hat{B} \cdot V_{o}}\right)
\end{gathered}
$$

em que $U_{o}$ e $V_{o}$ são, respectivamente, as amplitudes do deslocamento horizontal e vertical.

O ensaio para determinação do módulo dinâmico é, usualmente, realizado a várias freqüências com a finalidade de se formular uma curva módulo dinâmico $\times$ freqüência. No entanto, os equipamentos existentes possuem limitações para a aplicação destas freqüências de carregamento. Não obstante, fazendo-se uso do princípio da superposição tempo - temperatura (King, 2004), pode-se obter valores experimentais de módulo dinâmico em freqüências fora do limite de aplicação do equipamento. Tal princípio é aplicado a materiais ditos termo-reologicamente simples, ou seja, materiais em que a condição de temperatura transiente pode ser prevista a partir da resposta sob condições isotérmicas, ou seja

$$
\left|E^{*}\right|(\omega, T)=\left|E^{*}\right|(\widetilde{\omega})
$$

em que $T$ e $\widetilde{\omega}$ são, respectivamente, a temperatura e a freqüência reduzida. O módulo dinâmico, então, deixa de ser função da freqüência e temperatura, para depender, somente, da freqüência reduzida, determinada pela equação

$$
\begin{aligned}
\log (\widetilde{\omega}) & =\log \left(\omega \cdot a_{T}\right) \\
a_{T} & =\frac{\omega_{\text {Tref }}}{\omega}
\end{aligned}
$$

sendo $\omega_{\text {Tref }}$ e $\omega$, respectivamente, a freqüência da temperatura de referencia e a freqüência da temperatura em questão. $\mathrm{O}$ parâmetro $a_{T}$ é o único parâmetro dependente da temperatura, conhecido como fator de translação horizontal (horizontal shift factor) (Schapery, 1984). Pode ser determinado experimentalmente, porém, é necessário que as curvas de módulo dinâmico $\times$ freqüência sobreponham-se para temperaturas adjacentes. Esse parâmetro é determinado pela Equação 18 (tentativa inicial). Posteriormente, $\log \left|\mathrm{E}^{*}\right|$ é ajustado por meio de um método de minimização de erros, utilizando-se para o módulo dinâmico a função sigmoidal (Chehab, 2002)

$$
\log \left|E^{*}\right|=a+\frac{b}{1+\frac{1}{e^{d+g \cdot \log (\widetilde{\omega})}}}
$$

sendo $a, b, d$ e $g$ parâmetros a serem ajustados.

É importante observar que ao empregar a Equação 19 e os parâmetros $a_{T}$ (para as diferentes temperaturas) podem-se construir curvas módulo dinâmico $\times$ freqüência, concernentes a temperaturas onde não se possui dados experimentais (Momen, 2004). O modulo dinâmico pode, além disso, ser utilizado para obtenção do módulo de relaxação (domínio do tempo), segundo interconversão, conforme explicado anteriormente, com utilização da série de Prony

$$
E^{\prime}(\widetilde{\omega})=E_{\infty}+\sum_{i=1}^{M} \frac{\widetilde{\omega} \cdot \rho_{i}^{2} \cdot E_{i}}{\widetilde{\omega}^{2} \cdot \rho_{i}^{2}+1}
$$

sendo $E^{\prime}(\widetilde{\omega})=\left|E^{*}\right| \cdot \cos (\theta)$, e, juntamente com o método da colocação, determinar os coeficientes $\rho_{i}$ (amortecedores do modelo mecânico de Maxwell generalizado), e os coeficientes $E_{i}$ (molas do referido modelo) (Chehab, 2002; Gibson, 2006; Park e Schapery, 1999; Souza, 2004).

Os mesmos coeficientes calculados para a Equação 20 são usados na equação que descreve o módulo de relaxação do material (Gibson, 2006)

$$
E(t)=E_{\infty}+\sum_{i=1}^{M} E_{i} \cdot e^{\frac{-t}{\rho_{i}}}
$$

\section{MATERIAIS E DOSAGEM}

\subsection{Agregados, ligante e filer}

Para a produção dos agregados sintéticos de argila calcinada (ASAC) selecionou-se como matéria-prima um solo fino (PUC), com base em estudos anteriores desenvolvidos pelo Grupo de Geotecnia (GEOTEC) da Universidade Federal do Amazonas (UFAM) (Frota et al., 2004). Realizaram-se os procedimentos geotécnicos típicos à determinação da textura (ABNT NBR 7181) e dos limites de consistência (ABNT NBR 6459 e NBR 7180). Os resultados são apresentados na Tabela 1 . O solo PUC foi classificado como A7-5 (AASHTO/TRB) e CH (SUCS).

$\mathrm{O}$ processo de confecção dos agregados sintéticos (Figura 2) seguiu as seguintes etapas: a) homogeneização da amostra (Figura 2a), b) moldagem de massas de solo em formato cilíndrico (Figura 2b), c) corte por

Tabela 1. Composição textural e Limites de Consistência da amostra PUC

\begin{tabular}{ccccccc}
\hline Areia & Silte & Argila & LL & LP & IP & passa na \# 0,075mm \\
\hline $15 \%$ & $41 \%$ & $44 \%$ & $55 \%$ & $31 \%$ & $24 \%$ & $92,0 \%$ \\
\hline
\end{tabular}




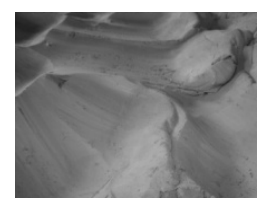

(a)

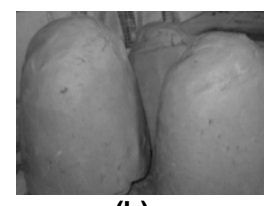

(b)

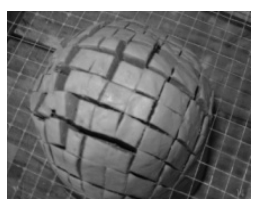

(c)

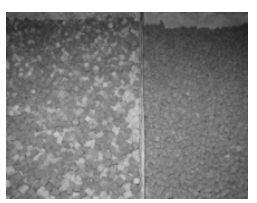

(d)

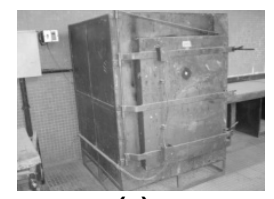

(e)

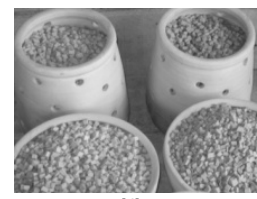

(f)

Figura 2. Processo de produção dos ASACs

meio de telas, conforme dimensão que se desejava para os agregados (Figura 2c), d) secagem à sombra (Figura 2d), e) calcinação, em forno elétrico, dos agregados acondicionados em jarros cerâmicos, à temperatura aproximada de $900^{\circ} \mathrm{C}$ (Figura 2e e 2f).

Produzidos os agregados sintéticos, os mesmos foram submetidos aos seguintes ensaios de qualificação, conforme estabelece a especificação do Departamento Nacional de Estradas de Rodagens (DNER), atual Departamento Nacional de Infra-Estrutura de Transportes (DNIT): a) Seleção Expedita pelo Processo de Fervura - Ensaio de Autoclave (DNER ME 223/94), b) Perda de Massa após Fervura (DNER ME 225/94) e, c) Desgaste por Abrasão "Los Angeles" (DNER ME 222/94). Conforme indica a Tabela 2 os resultados atenderam as especificações.

Tabela 2. Ensaios de qualificação dos ASACs

\begin{tabular}{lccc}
\hline \multicolumn{1}{c}{ Ensaio } & Norma & $\begin{array}{c}\text { Valores } \\
\text { Limites }\end{array}$ & Amostra \\
\cline { 4 - 4 } $\begin{array}{l}\text { Variação de textura e } \\
\text { forma }\end{array}$ & $\begin{array}{c}\text { DNER } \\
\text { ME }\end{array}$ & Não Varia & $\begin{array}{c}\text { Não } \\
\text { Varia }\end{array}$ \\
\hline $\begin{array}{l}\text { Perda de massa após } \\
\text { fervura }\end{array}$ & $\begin{array}{c}\text { DNER } \\
\text { ME }\end{array}$ & Máx 10\% & $0,14 \%$ \\
\hline $\begin{array}{l}\text { Desgaste por abrasão } \\
\text { "Los Angeles" }\end{array}$ & $\begin{array}{c}\text { DNER } \\
\text { ME }\end{array}$ & Máx 45\% & $43 \%$ \\
\hline
\end{tabular}

Na composição das mistura asfálticas foi empregado, além do ASAC, duas areias, sendo uma proveniente do município de Coari - AM (Areia 1) e a outra oriunda da região de Urucu - AM (Areia 2). Realizaramse análises granulométricas dos agregados de acordo com a norma ASTM C 136-95. Os ASACs, igualmente, foram avaliados, segundo a ASTM C 127-88, quanto: Apparent Specific Gravity (Gsa), Bulk Specific Gravity (Gsb) e Bulk Specific Gravity in the condition Saturated Surface Dry (Gsb ssd) e Absorption. Estes três últimos parâmetros, também, foram determinados para as areias (ASTM C 128-93). Caracterizaram-se, ainda, os agregados graúdos e miúdos, segundo: Loose Unit Weight (Wul) e Rodded Unit Weight (Wur), seguindo a norma AASHTO T-19/97. O
Cimento Asfáltico de Petróleo - CAP 50/70, fornecido pela Refinaria Isaac Sabbá (UN-Reman), foi classificado, conforme critério Superpave (SHRP, 1994a, 1994b), como PG 64-22. O cimento Portland (filer) apresentou massa específica real igual a $3,150 \mathrm{~g} / \mathrm{cm}^{3}$ (DNER ME 085/94) e, granulometria satisfazendo ao padronizado para material de enchimento pela norma DNER ME 367/97. A Tabela 3 indica esses resultados.

O material sintético, como já era esperado pelos estudos do Grupo de Geotecnia, por se tratar de agregado leve, apresentou sua densidade (Específica Aparente, Solta e Compactada) inferior às respectivas densidades das Areias ( 1 e 2), e um alto potencial de absorção $(20 \%)$. Tal resultado indica que a mistura composta por esse tipo de material necessitará de uma maior quantidade de ligante, em relação às misturas confeccionadas com agregados naturais, tendo-se em vista a absorção de parte do mesmo pelo ASAC (Nunes, 2006).

\subsection{Dosagem da mistura asfáltica}

A mistura granulométrica seguiu a Faixa Superpave, com Diâmetro Máximo Nominal (DMN) de 12,5 mm (SHRP, 1994a, 1994b). As porcentagens relativas aos agregados foram definidas de acordo com o Método Bailey, cujo procedimento de seleção é demonstrado em E-C044 (TRB, 2002). A Figura 3 mostra o enquadramento da curva granulométrica da mistura composta por: ASAC $(60,05 \%)$, Areia $1(19,68 \%)$, Areia 2 $(16,85 \%)$ e cimento Portland $(3,42 \%)$.

Determinou-se o teor ótimo de ligante segundo procedimento do $3^{\circ}$ Distrito Rodoviário Federal - $3^{\circ} \mathrm{DRF}$ (Soares, 2000), que se baseia nos valores de Volume de Vazios (Vv) e na Relação Betume - Vazios (RBV). Os valores obtidos para T1, T2, T3 e T4, foram, respectivamente, $6,57 \%, 5,79 \%, 6,35 \%$ e $6,79 \%$. O teor ótimo "T", definido pela média dos dois valores centrais, ou seja, pela média simples de T1 e T3, resultou no valor de $6,46 \%$ (Figura 4). O material sintético, por apresentar-se como agregado poroso, absorveu parte do ligante $(0,70 \%)$.

Tabela 3. Ensaios de caracterização dos agregados

\begin{tabular}{ccccccc}
\hline Amostra & $\begin{array}{c}\mathbf{G s b} \\
\left(\mathbf{g} / \mathbf{c m}^{3}\right)\end{array}$ & $\begin{array}{c}\mathbf{G s b} \text { ssd } \\
\left(\mathbf{g} / \mathbf{c m}^{3}\right)\end{array}$ & $\begin{array}{c}\mathbf{G s a} \\
\left(\mathbf{g} / \mathbf{c m}^{3}\right)\end{array}$ & $\begin{array}{c}\text { Absorption } \\
(\mathbf{\%})\end{array}$ & $\begin{array}{c}\text { Wul } \\
\left(\mathbf{k g} / \mathbf{m}^{3}\right)\end{array}$ & $\begin{array}{c}\text { Wur } \\
\left(\mathbf{k g} / \mathbf{m}^{3}\right)\end{array}$ \\
\hline ASAC PUC & 1,730 & 2,076 & 2,644 & 20,00 & 1021,25 & 1107,31 \\
\hline Areia 1 & 2,604 & 2,677 & - & 0,74 & - & 1710,82 \\
\hline Areia 2 & 2,601 & 2,674 & - & 0,73 & - & 1583,30 \\
\hline
\end{tabular}




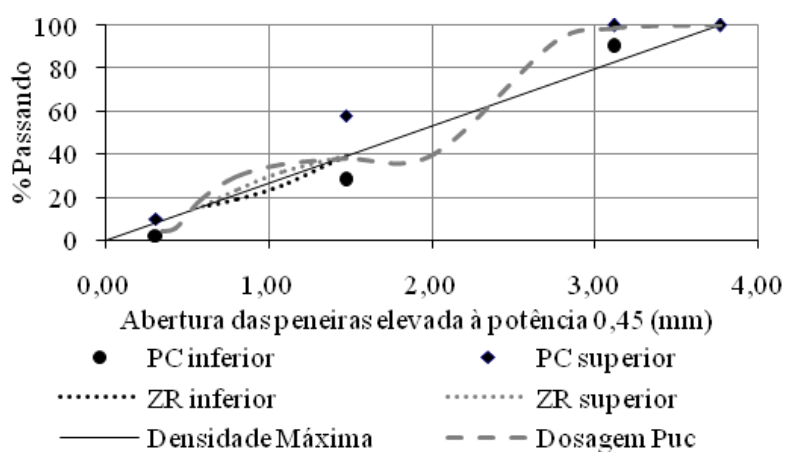

Figura 3. Curva granulométrica das misturas avaliadas

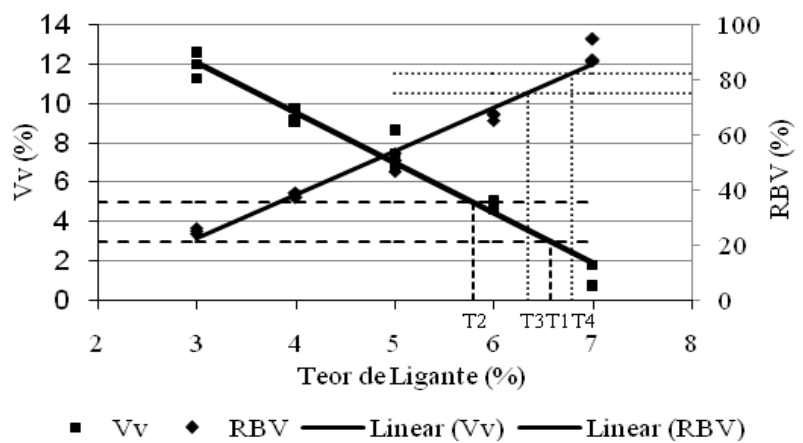

Figura 4. Determinação do teor ótimo de ligante pelo $3^{\circ} \mathrm{DRF}$

Os corpos de prova foram compactados pelo Método Marshall, com aplicação de 75 golpes por face (DNER ME 043-95). Antes da compactação, visando simular o efeito de envelhecimento de curto prazo, as misturas ficaram duas horas em estufa e à $10^{\circ} \mathrm{C}$ acima da temperatura de compactação (AASHTO PP-2). As temperaturas de preparo e compactação, definidas pelo Método de Ensaio DNER ME 004-94, ficaram compreendida entre $158^{\circ} \mathrm{C}$ e $167^{\circ} \mathrm{C}$ e $148^{\circ} \mathrm{C}$ e $157^{\circ} \mathrm{C}$, respectivamente. A Densidade Específica Máxima da mistura (Gmm) foi encontrada pelo Rice Test (ASTM D 2041), para um teor de ligante de $6.0 \%$, permitindose, assim, a determinação da fração de ligante absorvida pelos agregados ( $\mathrm{Pba}$ ), conforme critérios Superpave (SHRP, 1995). Segundo os mesmos critérios, encontrou-se a Gmm no teor inicial, sendo as demais densidades $(\mathrm{Gmm})$, concernentes aos outros teores, obtidas por meio de cálculo. O resultado para teor ótimo da mistura $(6,46 \%)$ foi de $1,912 \mathrm{~g} / \mathrm{cm}^{3}$.

\section{PROGRAMA EXPERIMENTAL}

$O$ ensaio de módulo dinâmico é normalizado pela AASHTO TP 62-03, onde prescreve que o mesmo deve ser realizado nas temperaturas de $-10^{\circ} \mathrm{C}, 4,4^{\circ} \mathrm{C}$, $21,1^{\circ} \mathrm{C}, 37,7^{\circ} \mathrm{C}$ e $54,4^{\circ} \mathrm{C}$, acarretando um experimento muito demorado (aproximadamente 48h). No entanto, estudos realizados pela North Carolina State University, sobretudo o trabalho de King (2004), mostram que existe uma superposição entre as curvas de módulo dinâmico, alusivas às temperaturas de $-10^{\circ} \mathrm{C}, 10^{\circ} \mathrm{C}$ e $35^{\circ} \mathrm{C}$, quando se aumenta a faixa de freqüência, levando a redução do tempo total de ensaio. A Tabela 4 indica as condições propostas na citada norma e aquelas sugeridas por King (2004).

Tabela 4. Parâmetros de ensaio para o Módulo Dinâmico

\begin{tabular}{ccc}
\hline Método & Freqüências (Hz) & Temperaturas $\left.^{\circ} \mathbf{C}\right)$ \\
\hline \multirow{2}{*}{ AASHTO TP 63-03 } & 0,$1 ; 0,5 ; 1,0 ;$ & $-10,0 ; 4,4 ; 21,1 ;$ \\
& $5 ; 10 ; 25$ & 37,$7 ; 54,4$ \\
\hline \multirow{2}{*}{ KING (2004) } & 0,$01 ; 0,05 ; 0,10 ;$ & \\
& 0,$5 ; 1,0 ; 5,0 ;$ & $-10 ; 10 ; 35$ \\
& $10 ; 25$ & \\
\hline
\end{tabular}

Neste artigo empregou-se o método indicado por King (2004), sendo os ensaios mecânicos realizadas em uma UTM (IPC Global) (Figura 5a) acoplada a uma câmara de temperatura BASS, com capacidade para atingir valores de $-15^{\circ} \mathrm{C}$ a $85^{\circ} \mathrm{C}$ (Figura $5 \mathrm{~b}$ ). Para medir os deslocamentos, ocorrentes nos corpos de prova, utilizaram-se micros LVDTs, colocados a uma distância de $50,8 \mathrm{~mm}$ entre si, nas direções vertical e horizontal (Figura 5c).

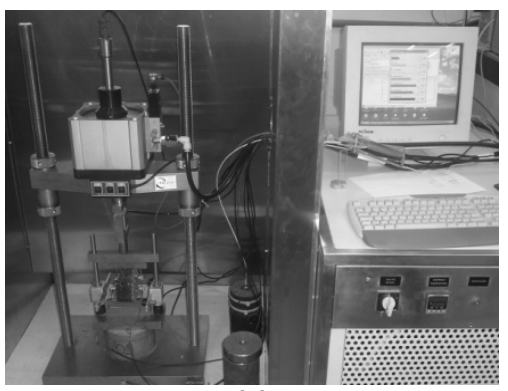

(a)

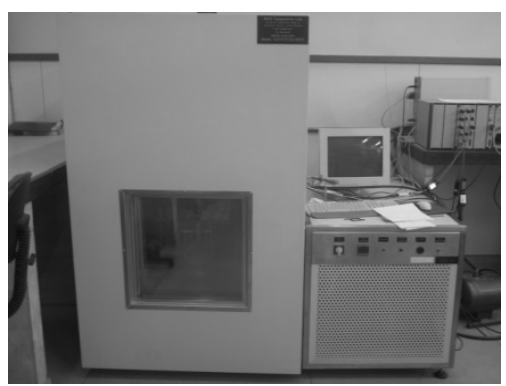

(b)

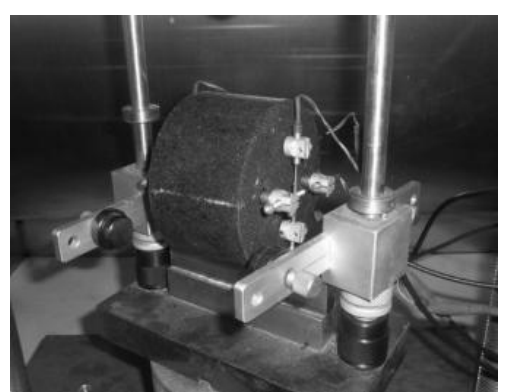

(c)

Figura 5. a) Máquina Universal de Ensaios, b) Câmara de temperatura, c) Montagem do CP 


\section{RESULTADOS}

Na Figura 6 tem-se um resultado típico do deslocamento medido no transcurso dos ensaios realizados. Como pode ser observado, existe um considerável nível de ruído nas leituras fornecidas pelos LVDTs. De modo a suavizá-las foi utilizada a função de distribuição normal de Gauss.

$$
y_{i}{ }^{\prime}=\frac{\sum_{j=1}^{n} k \cdot\left(\frac{x_{i}-x_{j}}{b}\right) \cdot y_{j}}{\sum_{j=1}^{n} k \cdot\left(\frac{x_{i}-x_{j}}{b}\right)}
$$

Sendo:

$$
k(f)=\frac{1}{0,37 \cdot \sqrt{2 \cdot \pi}} \cdot e^{-\frac{f^{2}}{2 \cdot(0,37)^{2}}}
$$

e $b$ é a largura de banda de $x$.

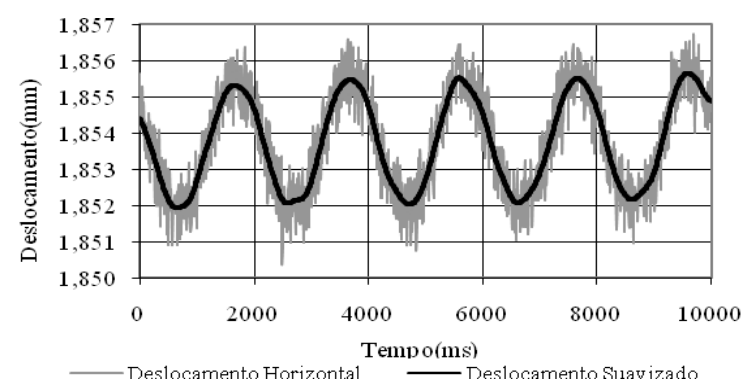

Figura 6. Deslocamentos medidos pelos LVDTs e suavização na temperatura de $35^{\circ} \mathrm{C}$

Após a suavização dos deslocamentos, os dados foram regredidos para funções na forma da Equação 24 (King, 2004; Chehab, 2002; Gibson, 2006)

$$
f(t)=a+b \cdot t+c \cdot \operatorname{sen}(\omega \cdot t+\varphi)
$$

sendo: $a, b, c$ e $\varphi$ foram parâmetros obtidos a partir da regressão. Utilizou-se a Equação 15 para o cálculo do módulo dinâmico, relativo a cada freqüência e temperatura. Em seguida, partindo-se das Equações 17, 18 e 19 construi-se a curva mestra.

As Figuras 7 e 8 apresentam, respectivamente, os resultados do deslocamento horizontal e vertical suavizados, de acordo com as equações 22 e 23 e, regredidos para a Equação 24. Observa-se que a mesma se enquadra muito bem aos dados experimentais suavizados. Na Figura 9 indicam-se os resultados concernentes ao módulo dinâmico, para as três temperaturas de ensaio.

Nota-se na Figura 9 que o valor do módulo dinâmico é diretamente proporcional à freqüência de aplicação da carga e inversamente proporcional à temperatura. Tais observações já eram aguardadas, uma vez que, para materiais viscoelásticos, como comentado anteriormente, o aumento da taxa de aplicação de carga aumenta sua rigidez.

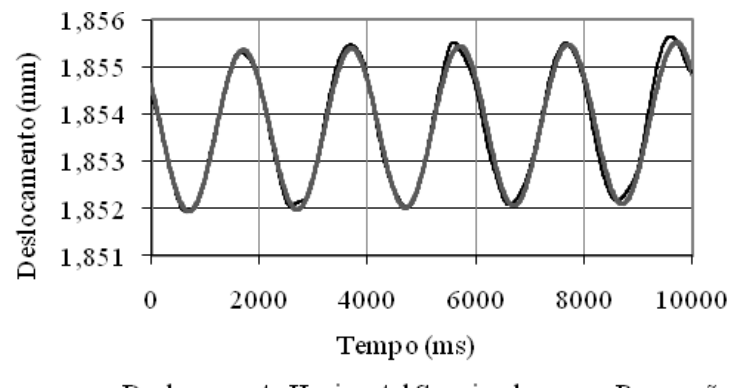

Figura 7. Regressão dos dados suavizados para o deslocamento horizontal e temperatura de $35^{\circ} \mathrm{C}$

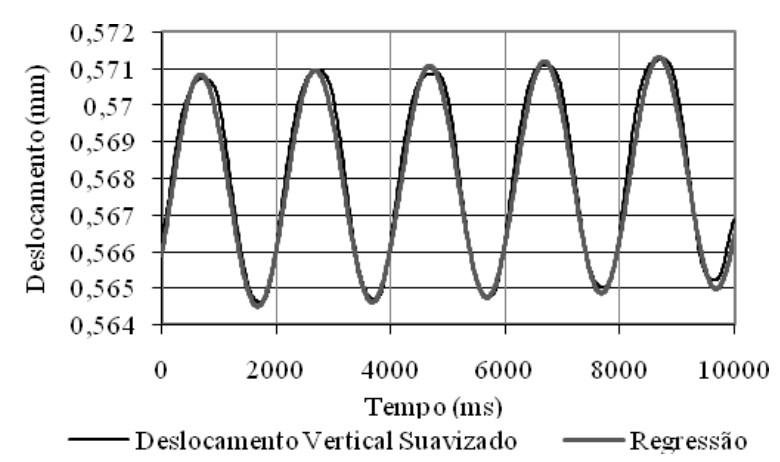

Figura 8: Regressão dos dados suavizados para o deslocamento vertical para temperatura $35^{\circ} \mathrm{C}$

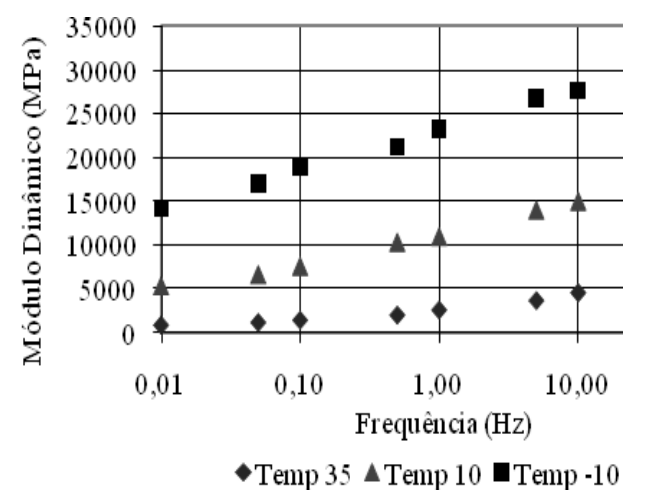

Figura 9. Módulo dinâmico para diferentes temperaturas de ensaio e freqüências de aplicação de carga

A obtenção experimental do fator de translação horizontal, de acordo com a Equação 19, só é possível se os valores do módulo dinâmico se sobrepuserem para temperaturas adjacentes. Na Figura 9 observa-se esta assertiva, verificando que o valor do módulo dinâmico é igual nas freqüências de $0.01 \mathrm{~Hz}$, para $10^{\circ} \mathrm{C}$, e na freqüência de $25 \mathrm{~Hz}$, para $35^{\circ} \mathrm{C}$. A Figura 10 mostra a curva mestra, considerando-se como $10^{\circ} \mathrm{C}$ a temperatura de referência. Tal curva foi utilizada na obtenção dos parâmetros da Equação 19 por um método de minimização de erros, sendo os fatores de translação horizontal ajustados posteriormente pela função polinomial (Equação 25). As Figuras 11 e 12 mostram, respectivamente, a função sigmoidal obtida e os fatores de translação horizontais para cada temperatura.

$$
\log a_{T}=a_{2} x^{2}+a_{1} x+a_{0}
$$




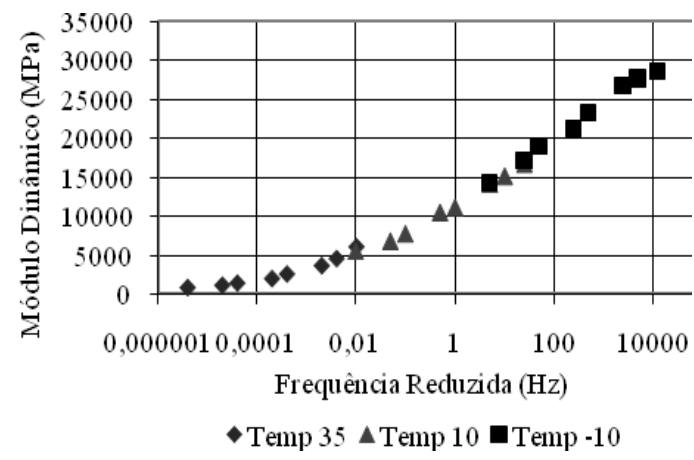

Figura 10. Curva mestra para o módulo dinâmico

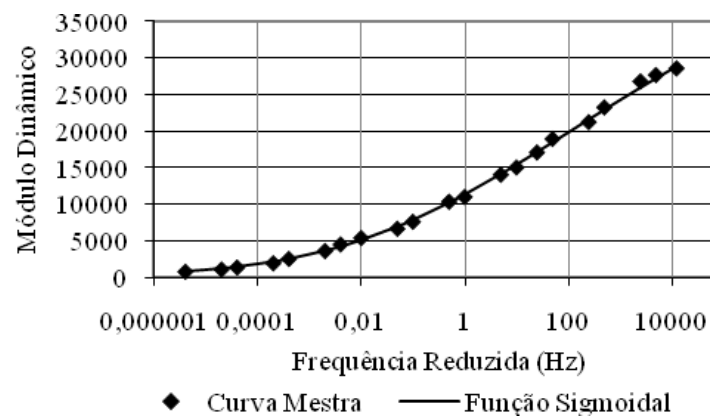

Figura 11. Dados experimentais $\times$ função sigmoidal regredida

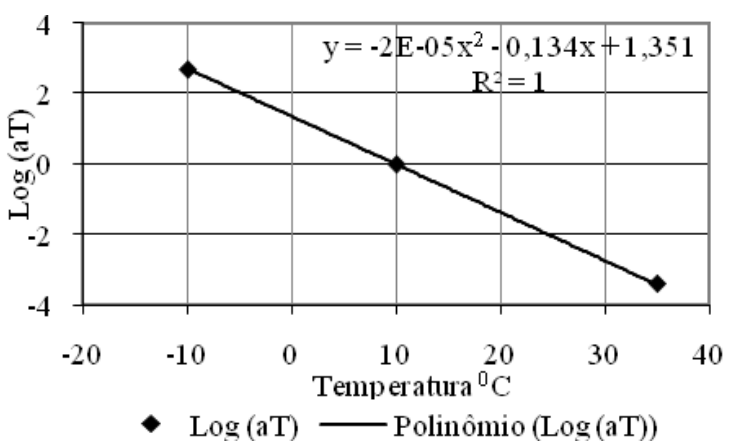

Figura 12. Fatores de translação horizontais para as temperaturas de ensaio

Na Tabela 5 têm-se os valores para os parâmetros atinentes à Equação 19, bem como aqueles obtidos para o polinômio de segundo grau, empregado na regressão dos fatores de translação horizontal. De posse desses parâmetros, pode-se calcular o valor do módulo dinâmico em qualquer freqüência e temperatura desejada.

A fim de validar os valores obtidos para este parâmetro (misturas confeccionadas com ASAC e utilizando o ensaio de compressão diametral), os mesmos foram comparados com os resultados de King (2004), realizados com ensaio de compressão axial (mistura densa com calcário e mistura aberta com granito), conforme apresentado na Figura 13. A Figura 14 indica os valores do módulo dinâmico, a partir dos parâmetros constantes na Tabela 5, para temperaturas onde não se possui dados experimentais.

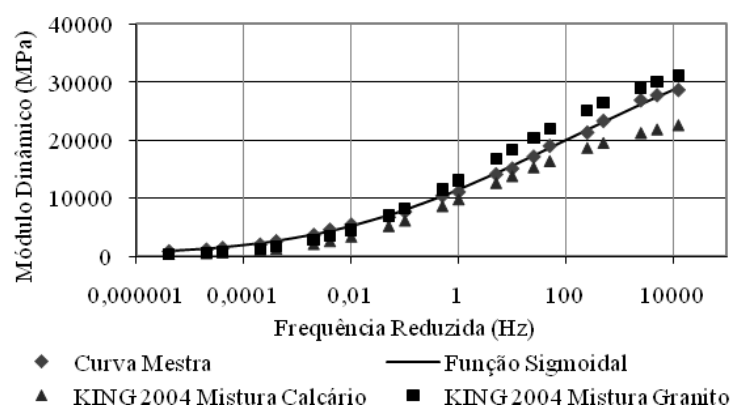

Figura 13. Comparação entre os resultados obtidos neste trabalho e os obtidos por KING (2004)

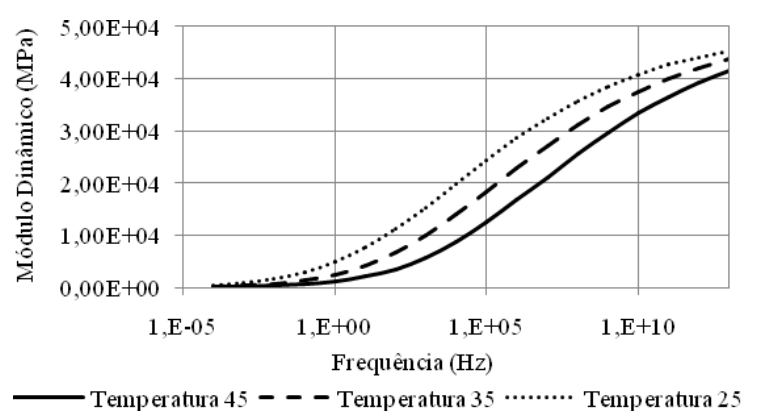

Figura 14: Módulo dinâmico obtido para diferentes temperaturas e freqüências a partir dos parâmetros constantes na Tabela 5

Observa-se, na Figura 14, que as curvas do modulo dinâmico obtidas, necessitam de altas freqüências para alcançarem o seu valor máximo. Este resultado já era esperado, porquanto foram empregadas altas temperaturas. Apesar disso, ao se observar à curva referente à temperatura de $35^{\circ} \mathrm{C}$ (temperatura ensaiada), tem-se que a mesma está em concordância com aquela apresentada na curva mestra (Figura 10).

\section{CONCLUSÕES}

Este trabalho focalizou a obtenção do módulo dinâmico para misturas asfálticas, utilizando-se o ensaio de compressão diametral em corpos de prova compactados nas dimensões $101,5 \times 63,50 \mathrm{~mm}$, parâmetro importante na caracterização dessas misturas, uma vez que é empregado como dado de entrada em modelos de previsão de vida de fadiga e deformação permanente. O mesmo pode também ser utilizado para a obten-

Tabela 5. Parâmetros de Regressão

\begin{tabular}{cccc|ccc}
\hline \multicolumn{4}{c}{ Função Sigmoidal $\left|E^{*}\right|$} & \multicolumn{3}{c}{ Função Polinomial $\boldsymbol{a}_{T}$} \\
\hline $\boldsymbol{a}$ & $\boldsymbol{b}$ & $\boldsymbol{d}$ & $\boldsymbol{g}$ & $\boldsymbol{a}_{2}$ & $\boldsymbol{a}_{1}$ & $\boldsymbol{a}_{0}$ \\
\hline \multirow{2}{*}{1,0323956} & 3,6591611 & 1,5606974 & 0,2795805 & $-2,00 \mathrm{E}-05$ & $-0,134$ & 1,351 \\
\hline
\end{tabular}


ção do módulo de relaxação das citadas misturas. Como explicado na fundamentação teórica, esse módulo é necessário para a realização de análises numéricas, à luz de modelos viscoelásticos lineares (dependente do tempo). Além disso, cumpre destacar: ( $a$ ) o resultado constante na Figura 13 corrobora que o ensaio de compressão diametral pode ser empregado na obtenção do módulo dinâmico para misturas asfálticas, facilitando estudos de campo, onde não é possível obter amostras para a realização de ensaios axiais; $(b)$ a equivalência entre os resultados para as misturas asfálticas com ASAC (segundo o ensaio de compressão diametral) com os resultados obtidos pelo trabalho de KING (2004), utilizado o ensaio de compressão axial. Contudo, a fim de validar o tipo de mistura analisada, denota-se indispensável efetuar o ensaio de módulo dinâmico no modo de compressão diametral para misturas com agregados convencionais; $(c)$ o método empregado neste artigo para a obtenção do módulo dinâmico diminuiu consideravelmente as temperaturas de ensaio, quando comparado com o método proposto pela AASHTO. Dessa maneira, as freqüências empregadas para suprir as temperaturas, onde não se tinham dados experimentais, foram suficientes para a sobreposição dos mesmos. Embora pertinente efetuar testes com outras espécies de misturas, as freqüências ensaiadas podem fornecer dados suficientes (sobreposição de dados) para qualquer tipo de mistura.

\section{REFERÊNCIAS BIBLIOGRÁFICAS}

AASHTO (1999) PP-2 - Standard Pratice for Mixture Conditioning of Mix Asphalt. American Association of State Highway and Transportation Officials. Washington, DC.

AASHTO (1997) T 19-93 - Standard Test Method for Unit Weight and Voids in Aggregate. American Association of State Highway and Transportation Officials . Washington, DC.

AASHTO (2003) TP 62-03 - Standard Method of Test for Determining Dynamic Modulus of Hot-Mix Asphalt Concrete Mixtures. American Association of State Highway and Transportation Officials. Washington, DC.

ABNT (1984) NBR 6459 - Solo: Determinação do limite de liquidez. Associação Brasileira de Normas Técnicas . Rio de Janeiro (1984).

ABNT (1984) NBR 7180 - Solo: Determinação do limite de plasticidade. Associação Brasileira de Normas Técnicas . Rio de Janeiro.

ABNT (1984) NBR 7181 - Solo: Análise granulométrica. Associação Brasileira de Normas Técnicas . Rio de Janeiro.

ABNT (1984) NBR 9937 - Grãos de solo que passam na peneira de \# 4,8mm: Determinação da massa específica. Associação Brasileira de Normas Técnicas . Rio de Janeiro.

ASTM (1988) C 127-88 - Standard Test Method for Specific Gravity and Absorption of Coarse Aggregate. American Society for Testing and Materials . Philadelphia, Pennsylvania.

ASTM (1993) C 128-93 - Standard Test Method for Specific Gravity and Absorption of Fine Aggregate. American Society for Testing and Materials . Philadelphia, Pennsylvania.

ASTM (1995) C 136-95 - Standard Test Method for Sieve Analysis of Fine and Coarse Aggregates. American Association of State Highway and Transportation Officials . Philadelphia, Pennsylvania.

ASTM (2000) D 2041 - Standard Test Method for Theoretical Maximum Specific Gravity and Density of Bituminous Paving Mixtures. American Society for Testing and Materials. Philadelphia, Pennsylvania.

Batista, F. G. (2004) Caracterização Física e Mecanística dos Agregados de Argila Calcinada Produzidos com Solos Finos da BR-163/PA.
Dissertação de Mestrado, Instituto Militar de Engenharia, Rio de Janeiro.

Chehab, G. (2002) Characterization of Asphalt Concrete in Tension Using a Viscoelastoplastic Model. Ph.D. Dissertation, North Carolina State University, Raleigh, NC.

DNER (1994) ME 004/94 - Material Betuminoso: determinação da viscosidade Saybolt-Furol a alta temperatura método da película delgada. Departamento Nacional de Infra-Estutura de Transportes. Rio de Janeiro, RJ.

DNER (1995) ME 043/95 - Misturas betuminosas a quente: Ensaio Marshall. Departamento Nacional de Infra-Estrutura de Transportes . Rio de Janeiro.

DNER (1994) ME 085/94 - Material finamente pulverizado: Determinação da massa específica real. Departamento Nacional de InfraEstrutura de Transportes . Rio de Janeiro.

DNER (1994) ME 222/94 - Agregado sintético fabricado com argila: Desgaste por Abrasão. Departamento Nacional de Infra-Estrutura de Transportes . Rio de Janeiro.

DNER (1994) ME 223/94 - Argilas para a fabricação de agregados sintéticos de argila calcinada: Seleção expedita pelo Processo de Fervura. Departamento Nacional de Infra-Etrutura de Transportes. Rio de Janeiro.

DNER (1994) ME 225/94 - Agregado sintético de argila calcinada: Determinação da Perda de Massa após Fervura. Departamento Nacional de Infra-Estrutura de Transportes . Rio de Janeiro.

DNER (1997) ME 367/97 - Material de enchimento para misturas betuminosas. Departamento Nacional de Infra-Estrutura de Transportes Rio de Janeiro.

Frota, C. A.; C. L. Alencar e C. P. Silva (2004) Influência do Tipo de Agregado na Qualidade Técnica de Misturas Asfálticas. Anais da $35^{a}$ Reunião Anual de Pavimentação, ABPv . Rio de Janeiro, RJ.

Frota, C. A.; C. L. Silva; M. G. R. Santos e R. V. Silva (2004) Caracterização de Solos para Obtenção de Agregados Sintéticos de argila calcinada. $35^{a}$ Reunião Anual de Pavimentação . Rio de Janeiro, RJ.

Gibson, N. H. (2006) A Viscoelastoplastic Continuum Damage Model for Compressive Behavior of Asphalt Concrete. Ph.D. Dissertation, North Carolina State University, Raleigh, NC.

Hondros, G. (1959) Evaluation of Poisson's Ratio and the Modulus of Materials of a Low Tensile Resistance by the Brazilian (Indirect Tensile) Test with Particular Reference to Concrete. Austr. J. Appl. Sci. , Vol. 10, No. 3, 243-268.

King, M. H. (2004) Determination of Dynamic Moduli in Uniaxial Compression for North Caroline Hot Mix Asphalt Concrete. Master Thesis, North Carolina State University, Raleigh, NC.

Momen, M. H. (2004) Complex Modulus Determination of Asphalt Concrete Using Indirect Tension Test. Master Thesis, North Carolina State University, Raleigh, NC.

BIBLIOGRAPHY $\backslash 11046$ Nunes, F. R. G. (2006) Caracterização Mecânica de Misturas Asfálticas com Agregados Sintéticos de Argila Calcinada Quanto à Deformação Permanente. Dissertação de Mestrado, Universidade Federal do Ceará, Programa de Mestrado em Engenharia de Transportes, Fortaleza, CE, Brasil.

Park, S. W. e R. A. Schapery (1999) Methods of Interconversion between Linear Viscoelastic Material Functions. Part I - A Numerical Method Based on Prony Series. International Journal of Solids and Structures, Vol. 36, pp. 1653-1675.

Schapery, R. A. (1984) Correspondence Principles and a Generalized Jintegral for Large Deformation and Fracture Analysis of Viscoelastic Media. International Journal of Fracture, Vol. 25, p. $195-$ 223.

SHRP (1994a) Superior Performance Asphalt Pavements (Superpave). The Product of SHRP Asphalt Research Program. Superpave Series $\mathrm{N}^{\circ}$. 1. Strategic Highway Research Program .

SHRP (1994b) Superior Performing Asphalt Pavements (Superpave): The Product of SHRP Asphalt Research Program. Superpave Series $\mathrm{N}^{\circ}$. 2. Strategic Highway Research Program .

Sides, A.; J. Uzan e M. Perl (1985) A comprehensive visco-elastoplastic characterization of sand-asphalt under compression and tension cyclic loading. ASTM Journal of Testing and Evaluation ( $\left.\mathrm{n}^{\mathrm{o}} 13\right)$, p. 59.

Soares, J. B.; F. A. Freitas e J. V. Branco (2000) Método de Previsão do Teor Ótimo de Ligante em Misturas Asfálticas Tipo CBUQ. $32^{a}$ Reunião Anual de Pavimentação, ABPv, Vol. I, p. 148-160 . Brasília, DF.

Souza, F. V. (2004) Modelo Multiescala para a Previsão da Evolução do Dano em Compósitos Viscoelásticos. Dissertação de Mestrado, 
Universidade Federal do Ceará, Programa de Mestrado em Engenharia de Transportes, Fortaleza, CE.

TRB (2002) Bailey Method for Gradation Selection in Hot-Mix Asphalt Mixture Design. Transportation Research Board of the National Academies, E-CIRCULAR 044 . Washington, DC.

Wen, H. (2001) Fatigue Performance Evaluation of WesTrack Asphalt Mixtures Based on Viscoelastic Analysis of Indirect Tensile Test. $\mathrm{Ph}$.D. Dissertation, North Carolina State University, Raleigh, NC.

Zhang, W. e A. Drescher (1997) Viscoelastic Analysis of Diametral Compression of Asphalt Concrete. Journal of Engineering Mechanics, Vol. 123, no. 6 